\title{
FR I JETS IN SOUTHERN RADIO GALAXIES
}

\author{
PAUL A. JONES AND BEN D. LLOYD \\ University of Western Sydney, Nepean, PO Box 10, Kingswood, \\ NSW, 2747, Australia
}

\section{Introduction}

Sources flagged as extended or multiple in the Molonglo Reference Catalogue (MRC, Large et al. 1981, 1991), south of $\delta=-30^{\circ}$, were observed with the Molonglo Observatory Synthesis Telescope (MOST) with a resolution of 44 arcsec at $843 \mathrm{MHz}$ (Jones and McAdam 1992) to give a sample of 193 southern extended sources. Optical identifications were made using the UKST $b_{\text {J }}$ sky survey. We are now using the Australia Telescope Compact Array (ATCA) near Narrabri in Australia to study a subsample of Fanaroff-Riley class I radio galaxies and fit models to the jets.

We use two simultaneous wavelength bands $(20 / 13 \mathrm{~cm}$ or $6 / 3 \mathrm{~cm})$ and measure full polarisation with the ATCA, with its 6 antennas in a $6 \mathrm{~km}$ array and for some sources use several 12-hour observations including the $1.5 \mathrm{~km}$ configurations for extra shorter uv-spacings. We are also using the MOST data for the largest scales and best surface brightness sensitivity.

\section{MOST and ATCA results}

The MOST observations of the head-tail radio galaxy B1610-605 (Jones and McAdam 1992, 1994) show that the tail extends 26 arcmin at low surface brightness with width $<1$ arcmin. The neighbouring radio galaxy B1610-608 is a wide-angle-tail with the $W$ jet kinked towards the south 1 arcmin from the core. Both galaxies are in the nearby $(z=0.0143)$ Abell cluster A3627 (Abell et al. 1989). We have observed the two galaxies with the ATCA at 20 and $13 \mathrm{~cm}$ to study the tail, jets and lobes and how they interact with the intra-cluster medium (Jones and McAdam 1994, 1995). The tail of B1610-605 is narrow ( $<10$ arcsec within the first arcmin) so that if there are twin FR I jets from the core bent back by ram pressure, this must occur over the scale of a few kpc, within the optical galaxy. 
The brightness and width of the tail or jets were fitted and deconvolved using the model of a cylinder of Gaussian profile (Killeen et al. 1986). The standard assumptions for synchrotron emitting plasma were used to estimate the magnetic field and minimum pressure. The pressure profiles suggest that the intra-cluster medium is clumpy and that the cluster is not relaxed. This is confirmed by the ROSAT all-sky survey X-ray data ( $\mathrm{H}$. Böhringer, private communication).

The radio galaxy B1343-601 (Centaurus B) is one of the brightest extragalactic sources in the sky (240 Jy at $408 \mathrm{MHz}$ ), however there has been very little study of this galaxy because of its position (a) close to the Galactic plane (alternative name G309.6+1.7) and (b) in the far southern sky. The galaxy was optically identified by Laustsen et al. (1977) and its redshift measured as 0.01215 by West and Tarenghi (1989). The MOST observations (McAdam 1991) show prominent FR I jets and a diffuse, low surface brightness halo. We have recently (Sept/Oct 1995) observed this galaxy with the ATCA at 6 and $3 \mathrm{~cm}$ with two 12-hour observations, to allow detailed analysis and model-fitting of the jets.

Four galaxies which have prominent FR I jets, B1234-723, B1318-434, B1452-517 and B2148-555, selected from Jones and McAdam (1992), were observed with the ATCA at 6 and $3 \mathrm{~cm}$. Fits to the brightness, width, transverse position and polarisation of the jets at the two frequencies are used to determine the equipartition parameters and Bicknell's model for dissipative, turbulent FR I jets (Bicknell 1994) applied. The model takes a spline fit to the jet width and the derived pressure distribution and then predicts the surface brightness, Mach number, velocity, jet density ratio and mass flux. B1318-434 is identified with the elliptical galaxy NGC 5090 which is interacting with the spiral NGC 5091, and is discussed in more detail in Lloyd et al. (1995).

\section{References}

Abell G.O., Corwin H.G. and Olowin R. (1989), Astrophys. J. Suppl., 70, 1

Bicknell G.V. (1994), in Bicknell G.V., Dopita M.A. and Quinn P.J., eds, The First Stromlo Symposium: The Physics of Active Galaxies, ASP Conf. Ser. 54, Astronomical Society of the Pacific, p 357

Jones P.A. and McAdam W.B. (1992), Astrophys. J. Suppl., 80, 137

Jones P.A. and McAdam W.B. (1994), Proc. Astron. Soc. Australia., 11, 74

Jones P.A. and McAdam W.B. (1995), submitted to Mon. Not. Royal Astron. Soc.

Killeen N.E.B, Bicknell G.V. and Ekers R.D. (1986), Astrophys. J., 302, 306

Large M.I., Mills B.Y., Little A.G., Crawford D.F. and Sutton J.M. (1981), Mon. Not. Royal Astron. Soc., 194, 693

Large M.I., Cram L.E. and Burgess A.M. (1991), Observatory, 111, 72

Laustsen S., Schuster H.-E. and West R.M. (1977), Astron. and Astrophys., 59, L3

Lloyd B.D., Jones P.A. and Haynes R.F. (1995), Mon. Not. Royal Astron. Soc. in press McAdam W.B. (1991), Proc. Astron. Soc. Australia., 8, 255

West R.M. and Tarenghi M. (1989), Astron. and Astrophys., 223, 61 